\title{
Segmentation Of RT3D Ultrasound With Implicit Deformable Models Without Gradients
}

\author{
Elsa Angelini, Jeffrey Holmes, Andrew Laine, \\ Columbia University, \\ Department of Biomedical Engineering \\ ea179,jh553,al418@columbia.edu
}

\author{
Shunichi Homma \\ Columbia University, \\ Department of Medicine \\ sh23@columbia.edu
}

\begin{abstract}
This paper presents the implementation and validation of a new 3D deformable model method, based on the Mumford-Shah functional for segmentation of threedimensional real-time ultrasound. An experiment on 10 patients with primary hypertension was carried out to compare three segmentation methods for quantification of right and left ventricular ejection fraction: (1) manual tracing by an expert cardiologist, (2) 2D parametric deformable model, and (3) $3 D$ implicit deformable model implemented with a level set framework. Deformable model segmentations were performed on denoised data using a (3D+Time) brushlet expansion. The clinical study showed superior performance of the deformable model in assessing ejection fraction when compared to MRI measures. It also showed that the three-dimensional deformable model improved EF measures, which is explained by a more accurate segmentation of small and convoluted ventricular shapes when integrating the third spatial dimension.
\end{abstract}

\section{Introduction}

The challenge of developing a segmentation tool for quantification of cardiac function from threedimensional ultrasound lies in the wealth of dynamic information that can be extracted despite its relatively low spatial resolution and high level of speckle noise. We have been specifically interested in the use of realtime three-dimensional ultrasound data acquired with matrix-phased array transducers [1]. The poor quality of the ultrasound signal has limited the acceptance of this ultrasound technology in clinical practice, despite the wealth of information acquired by this system, far greater than with any other existing echocardiography screening modality. The work presented in this paper aimed to improve the better acceptance of this new technology by addressing the problem of automatic quantification of ventricular function.

Implicit deformable models, based on level set numerical methods [2] have been applied to a wide range of medical screening modalities for anatomical volume segmentation, shape representation and deformation modelization as reviewed in [3-5]. These deformable models rely on external forces derived from gradient information to stop the curve evolution at edge boundaries. A different approach was recently proposed by Chan and Vese [6] using a stopping term based on Mumford-Shah segmentation technique [7] rather than a traditional gradient functional. Such a deformable model might be best suited for 3D ultrasound images where the myocardium wall is depicted as a bright textured object without well defined borders. In addition, this segmentation method combines the following advantages: (1) Arbitrary initialization of the object anywhere in the image, (2) Topology adaptation for multi-object segmentation, (3) No constraints on curve smoothness, (4) Self-adaptation for inward and outward flows.

\section{Method}

\subsection{Energy Functional}

Given an image $I$ and a curve $C$, Chan and Vese introduced the following energy functional as a modification of the Mumford-Shah minimal partition problem [7]:

$$
\begin{aligned}
& E\left(C, c_{0}, c_{1}\right)=\mu L(C)+\nu A(C)+ \\
& \quad \lambda_{0} \int_{\text {inside }(C)}\left|I_{0}-c_{0}\right|^{2} d \Omega+\lambda_{1} \int_{\text {outside }(C)}\left|I_{0}-c_{1}\right|^{2} d \Omega
\end{aligned}
$$

where $c_{0}$ and $c_{1}$ are equal to the average of $I$ inside and outside of the curve $C, L(C)$ measures the length and $A(C)$ the area of the curve $C$, and $\left(\mu, \nu, \lambda_{0}, \lambda_{1}\right)$ are real scalar positive parameters.

\subsection{Level set Implementation}

Energy minimization of Equation (1) was performed with the level set framework introduced by Osher and Sethian [8]. Applying the level set framework to the problem of minimization of the energy in Equation (1), the curve $C$ is embedded in a Lipschitz scalar function $\phi$ defined on $\mathbb{R}^{N}$, such that:

$$
\begin{aligned}
& C=\left\{x \in \mathbb{R}^{N} / \phi(x)=0\right\}, \\
& C_{\text {inside }}=\left\{x \in \mathbb{R}^{N} / \phi(x)<0\right\}, \\
& C_{\text {outside }}=\left\{x \in \mathbb{R}^{N} / \phi(x)>0\right\} .
\end{aligned}
$$

The level set implementation of the energy functional replaces the n-D curve $C$ by an n+1-D function $\phi$ and solves the minimization problem so that the curve $C_{0}$ that minimizes the energy functional corresponds to the 
zero-level of the final solution $\phi_{0}$ of the level set framework. To rewrite the functional of Equation (1) as a function of $\phi$, two mathematical functions are used to define the interior and the contour of the curve $C$ :

(1) a Heaviside function: $H(z)=\left\{\begin{array}{l}1 \text { if } z \leq 0 \\ 0 \text { if } z \geq 0\end{array}\right.$,

(2) a Dirac function: $\delta(z)=\frac{d H(z)}{d z}$.

The regularization terms $L(C)$ and $A(C)$ can then be expressed in terms of $\phi$ and the energy functional of Equation (1) is rewritten as:

$$
\begin{aligned}
& E\left(\phi, c_{0}, c_{1}\right)=\mu L(\phi=0)+\nu A(\phi \leq 0)+ \\
& \lambda_{0} \int_{\Omega}\left|I-c_{0}\right|^{2} H(\phi) d \Omega+\lambda_{1} \int_{\Omega}\left|I-c_{1}\right|^{2}(1-H(\phi)) d \Omega
\end{aligned}
$$

\subsection{Dynamic Implementation}

Computation of the Euler-Lagrange equation associated with the minimization of Equation (5) requires regularization of the Heaviside and Dirac functions [6, 9]. In our implementation, we have chosen the regularization functions $H_{\varepsilon}(\phi)$ and $\delta_{\varepsilon}(\phi)$ proposed by Chan et al. in [6].

The Euler-Lagrange equation, as derived in [6], is expressed as:

$$
\begin{aligned}
& \delta_{\varepsilon}(\phi)\left[\mu \operatorname{div}\left(\frac{\nabla \phi}{|\nabla \phi|}\right)-\nu-\lambda_{0}\left(I-c_{0}\right)^{2}+\lambda_{1}\left(I-c_{1}\right)^{2}\right]=0 \\
& \frac{\delta_{\varepsilon}(\phi)}{|\nabla \phi|} \frac{\partial \phi}{\partial n}=0 \text { on } \partial \Omega,
\end{aligned}
$$

where $\frac{\partial}{\partial n}$ defines the partial derivative along the normal to the zero-level curve of $\phi$. We observe here the introduction of the curvature of the zero-level front $K(\phi)=-\operatorname{div}\left(\frac{\nabla \phi}{|\nabla \phi|}\right)$, as with the popular level set algorithm for segmentation via motion under mean curvature introduced by Osher and Sethian [8].

\subsection{Discretization and Parameters Values}

Implementation was performed with a finite difference scheme for spatial derivatives and the introduction of an artificial time component to model the descent iteration. Using an explicit scheme for the time derivative, the iterative process is written as:

$$
\phi_{i, j, k}^{n+1}=\phi_{i, j, k}^{n}+\Delta t^{*} \delta_{\varepsilon}\left(\phi_{i, j, k}^{n}\right)\left[\begin{array}{l}
-\mu d i v\left(\frac{\nabla \phi_{i, j, k}^{n}}{\left|\nabla \phi_{i, j, k}^{n}\right|}\right)+\nu+ \\
\lambda_{0} \frac{\left(I_{i, j, k}-c_{0}\left(\phi^{n}\right)\right)^{2}}{c_{0}\left(\phi^{n}\right)^{2}}- \\
\lambda_{1} \frac{\left(I_{i, j, k}-c_{1}\left(\phi^{n}\right)\right)^{2}}{c_{1}\left(\phi^{n}\right)^{2}}
\end{array}\right] .
$$

with $\lambda_{0} \geq 0, \lambda_{1} \geq 0, \mu \geq 0, \nu \geq 0$. We point out here that the signs were modified from the original implementation of Chan et al. [6], as we considered $\phi<0$ inside the object to be consistent with the standard level set implementation in [10]. We also modified the terms associated with the average values to better control their effect on the deformation process through parameters $\lambda_{0}$ and $\lambda_{1}$.The curvature term was computed with the mean curvature model, following the initial approach of Malladi, Sethian and Vemuri [10] for motion under mean curvature. The spatial derivatives were approximated with centered differences. We point out here that Chan et al. [6] used a different curvature model initially proposed by Rudin, Osher and Fatemi in [11], which combined both centered and one-sided spatial derivatives but did not explicitly include cross derivatives. They also used an implicit scheme for the curvature term to constrain bounds on $\phi^{n}$ and $\phi^{n+1}$ to be similar [12] and make the numerical scheme unconditionally stable. Unconditional stability allows for arbitrary time step values to speed up the iteration process. On the other hand, the semi-implicit scheme requires two updates of the function $\phi^{n+1}$ at each iteration, which can become problematic when manipulating large volumetric data sets. In our implementation we sacrificed some stability but gained implementation simplicity and computation speed, while using small time steps to keep the iterative process stable.

Parameters were set to the following values. We set $v=0$ to eliminate the constant term in Equation (6), which was derived from the area term in Equation (1). This parameter can be seen as an advection term that should be turned on only if we want to push the model in a constant direction (outward or inward). Here, we wanted the model to be able to move in any direction, allowing more arbitrary position in the initialization. We also set $\lambda_{1}=0$ so that we are not looking for a dual segmentation of the myocardium and the ventricular cavity as two homogeneous areas but rather for a single segmentation of the cavity. The myocardium tissue is rather inhomogeneous and the average intensity term $c_{1}(\phi)$ associated to this parameter led to unstable behavior of the model when not set to zero. The other parameters were set experimentally to $\quad \lambda_{0}=0.25, \mu=1$, and $\varepsilon=2$. 
Spacing steps were set to $\Delta x=\Delta y=\Delta z=1$. The time step was set to $\Delta t=\frac{\min (\Delta x, \Delta y, \Delta z)}{\left(|\mu|+|\nu|+\left|\lambda_{0}+\lambda_{1}\right|\right)}$ to ensure stability of the numerical scheme via CFL condition.

\subsubsection{Reinitialization}

When working with level sets and Dirac functions, it is necessary to reinitialize the function $\phi^{n}$ as the signed distance function to its zero-level. This procedure prevents the function $\phi^{n}$ from becoming too flat and ensures that the iterative process will converge to a minimum. This reinitialization is problematic in standard level set implementations where the zero-level is generally explicitly computed [10] and distance is computed for each point on the grid to the set of zerolevel points. This procedure is computationally expensive and very inefficient. A fast marching method has been proposed by Sethian [13] to reduce the computation time. An alternative to this approach was proposed by Osher in [14] via solving an iterative process to "straighten out" the function on either side of the zero level.

\subsection{Principle Steps of the Algorithm}

The sequence of function $\phi^{n}$ for $n \geq 0$ is constructed as follows:

1. Start with $n=0$. Initialize with a set of points a curve $C_{0}$ in the image domain. Define $\phi^{0}$ as the distance function with zero-level defined at the location of the curve $C_{0}$.

2. Compute $c_{0}\left(\phi^{n}\right)$ and $c_{1}\left(\phi^{n}\right)$ as the average of the function $\phi^{n}$ on the domains defined by $\phi^{n}<0$ and $\phi^{n}>0$ respectively.

3. Compute the curvature term for $\phi^{n}$.

4. Solve Equation (7) to obtain $\phi^{n+1}$.

5. Reinitialize $\phi^{n+1}$ as the signed distance function.

6 . Check whether the solution is stationary. Iterate for $n=n+1$ if not.

\section{Experiments}

We present in this section the methods and results for an in-vivo study performed to validate our segmentation method for quantification of left and right ventricular function in patients diagnosed with pulmonary hypertension (PH) [15]. This condition results in an increase in RV volume affecting the pumping function on the right side.

Quantification of ventricular volume from standard two-dimensional echocardiography is commonly performed via planar measurements and use of a geometric model for volume estimation. The left ventricle has an ellipsoidal shape with strong symmetry along a central axis, which allows volume estimation from 2D echocardiographic images using geometrical models. On the other hand, the right ventricle is crescent shaped and does not follow and axis of symmetry. For this reason, right ventricular volume cannot be estimated from planar views and its function is not well quantified during an echocardiographic examination. In such situations, RT3D ultrasound appears to be a very well suited screening tool as it can acquire the entire RV cavity in real-time to visually depict anatomical changes and quantify LV and RV cardiac function for early diagnosis and accurate assessment of the severity of the pathology.

\subsection{Study Protocol}

To test this hypothesis we compared LV and RV volume and ejection fraction measures with RT3D ultrasound and MRI for a set of 10 volunteering patients with pulmonary hypertension. Typical RT3D volumes acquire $(64 \times 64)$ pixels in short axis and contained 300 slices in the long axis dimension with a slice thickness of $0.308 \mathrm{~mm}$. Between 10 and 17 frames were acquired per cardiac cycle.

\subsubsection{Denoising with Brushlet Expansion}

Denoising was performed with a spatio-temporal brushlet expansion as described in [16, 17]. Brushlet analysis was performed in 3D for spatial denoising, followed by temporal denoising in 1D and (2D+Time) for enhancement of cardiac structures. Tiling of the Fourier domain was limited to four sub-intervals in each direction due to the low frequency harmonics of cardiac wall motion.

\subsubsection{Manual tracing on RT3D data}

An expert cardiologist manually traced endocardial contours on selected slices and computed ventricular volumes via sector plane-disks summation using the same dedicated software package from 3Dechotech $\subset$. Manual tracing was performed on the original data.

\subsubsection{Manual tracing on MRI data}

The MRI data was acquired with a $1.5 \mathrm{~T}$ clinical scanner using a standard cardiac MR protocol. The slice thickness was $8 \mathrm{~mm}$, with no gap in between. Acquisition was performed at a rate of 40-50 milliseconds per frame. An expert radiologist manually segmented the entire set of 10-15 two-chamber views acquired for each patient using a dedicated software display package installed in the department of Radiology at Columbia-Presbyterian Medical Center. For the two modalities, trabeculations and papillary muscles were excluded while moderator bands were included inside the cavity.

\subsubsection{Two-Dimensional Deformable Model Segmentation}

A two-dimensional parametric deformable-model segmentation was performed on the denoised data following the method described in [16]. The model was initialized with a 5 pixel-radius circle inside the cavity to segment. Next, the user manually selected the centers of the circle for every 10 slices. Center locations for in- 
between slices were determined via linear best fit. By identifying the best linear fit to the center points detected inside the LV cavity we defined an approximation of the $\mathrm{LV}$ medial axis.

\subsubsection{Three-Dimensional Deformable Model Segmentation}

Three-dimensional deformable-model segmentation was performed on the denoised data. The model was initialized similarly to the two-dimensional case, by manually selecting a center position inside the cavity to segment. But, contrary to the two-dimensional case, we did not need to define the model as entirely inside the cavity nor did we have to initialize it on every slice to segment. On the other hand, the Mumford-Shah functional was less stable than the two-dimensional balloon model as it converged towards the myocardium wall borders. We decided to initialize the model as a cylinder with ellipsoidal cross-section. The center and dimension of the ellipse were set up manually for each volume within the middle slice between the base and the apex of the ventricle. The ellipse dimensions were defined to reflect the ratio of dimensions of the ventricle, but the ellipse itself did not need to be close to the borders of the ventricle. We observed that using an ellipsoidal cross-section instead of a circle as for the two-dimensional model improved the stability and convergence of the iterative process. The cylinder height was defined from the base through 20 slices before the apex. By not including the apex we let the model deform in the third dimension from slices above that have better resolution and we were able to avoid problems of instability and over segmentation as encountered in the two-dimensional case.

\subsubsection{Study results}

The set of ten patients used in this study presented great diversity in the quality of the data as well as the shapes and sizes of the ventricles. Volume ranges for this study were the following: RV-ESV 71.5-269 ml, RV-EDV 123.03-311.5 ml, LV-ESV 19.0-82.2 ml, LV-EDV 41.5-144.8 ml, RV EF 9.1-51.6 \% and LV EF 30.4-74.5 $\%$ as obtained with manual tracing on MRI and RVESV 49.2-191.3 ml, RV-EDV 96.5-250.6 ml, LV-ESV 11.8-50.8 ml, LV-EDV 35.8-78 ml, RV-EF 44.2-68.1 $\%$ and LV-EF 40.8-68.1\% as obtained with manual tracing on RT3D ultrasound.

Absolute errors of measures were computed for RV and LV ejection-fraction. We evaluated mean-error values and standard deviation over the ten cases for the two ventricles. These error measures are reported in Table 1 and Table 2. Maximum and minimum error values are provided to better assess the range of variability achieved by the different segmentation methods.

For both ventricles, ejection fraction estimation was better estimated with the deformable model. For each segmentation method, measurement accuracy was lower for the right ventricle. This result could be explained by the fact the right ventricular volumes were significantly bigger than the left ventricular volumes as higher quantification errors for bigger volumes was also reported in similar study using RT3D ultrasound [18].

\begin{tabular}{|c|c|c|c|}
\hline $\begin{array}{c}\text { Error } \\
\text { (EF \%) }\end{array}$ & $\begin{array}{c}\text { Manual } \\
\text { vs. MRI }\end{array}$ & $\begin{array}{c}\text { 2D } \\
\text { Deformable } \\
\text { model vs. } \\
\text { MRI }\end{array}$ & $\begin{array}{c}\text { 3D } \\
\text { Deformable } \\
\text { model vs. } \\
\text { MRI }\end{array}$ \\
\hline Mean & 8.6 & 4.9 & 4.6 \\
\hline Std & 5.7 & 4.1 & 4.2 \\
\hline Max & 17.8 & 12.21 & 13.9 \\
Min & 0.3 & 0.2 & 0.8 \\
\hline
\end{tabular}

Table 1: Absolute errors in quantification of RV ejection fraction for ten clinical patient studies.

\begin{tabular}{|c|c|c|c|}
\hline $\begin{array}{c}\text { Error } \\
\text { (EF \%) }\end{array}$ & $\begin{array}{c}\text { Manual } \\
\text { vs. MRI }\end{array}$ & $\begin{array}{c}\text { 2D } \\
\text { Deformable } \\
\text { model vs. } \\
\text { MRI }\end{array}$ & $\begin{array}{c}\text { 3D } \\
\text { Deformable } \\
\text { model vs. } \\
\text { MRI }\end{array}$ \\
\hline Mean & 10.8 & 7.1 & 4.4 \\
\hline Std & 5.3 & 4.5 & 5.1 \\
\hline Max & 18.4 & 16.1 & 18.2 \\
Min & 3.2 & 0.3 & 0.1 \\
\hline
\end{tabular}

Table 2: Absolute errors in quantification of $\mathrm{LV}$ ejection fraction for ten clinical patient studies.

To assess the agreement between the different methods, we first performed a linear regression to evaluate the correlation between the segmentation methods and to determine the statistical significance of our measurements. Regression plots for RV and LV EF measurements are displayed in Figure 1. Regression statistics with p-values for [LV; RV] ejection fraction were: $p=[0.23 ; 0.062]$ when evaluated with manual tracing on RT3D ultrasound, $p=[0.0009 ; 0.0008]$ when evaluated with 2D deformable model on RT3D ultrasound and $p=[0.0008 ; 0.0006]$ when evaluated with 3D deformable model on RT3D ultrasound.

We observed again an improvement in correlation with the deformable model when compared to manual tracing. In the case of manual tracing, the weak correlation could be explained by the abnormal LV shapes, due to the enlargement of the RV cavity in $\mathrm{PH}$ patients. Volume measurement with manual tracing on RT3D relies on geometrical models that assume a particular smoothness of its contours. This assumption is generally true in normal patients, but will introduce measurement errors in cases of disease such as $\mathrm{PH}$ where abnormal irregular shapes are encountered due to high pressure in the RV cavity, compressing the LV and introducing deformation on the septal side. On the other hand, the high pressure in the RV induces a change in the ventricular shape, becoming rounder as it grows in size, and correlations for this ventricle were similar to values obtained for the LV in prior clinical studies [16]. Measures for the RV were more significant than for the $\mathrm{LV}$ with both segmentation methods, as the p-value for this ventricle were lower. Overall, deformable model correlation coefficients were similar for both studies but p-values with the deformable model were greatly improved when compared to the first study. This better 
agreement via linear regression reflects the superior performance of the deformable model to accurately segment the ventricular volumes on denoised RT3D data. Finally, the 3D deformable model improved segmentation accuracy for both correlation and $p$-value when comparing to $2 \mathrm{D}$ implementation. This result is in accordance with our expectations that 3D deformable models should perform better than their 2D counterpart by integrating spatial continuity in all three spatial dimensions.

Similar clinical studies have been reported recently in the clinical literature. Corsi et al. [18] performed a study for LV EF measurement with MRI and 3D level set segmentation on RT3D data. They reported a correlation coefficient of $r=0.98$ (MRI vs. level set) and $\mathrm{r}=0.99$ (MRI vs. manual tracing). The study included 21 patients and no significance levels were reported.

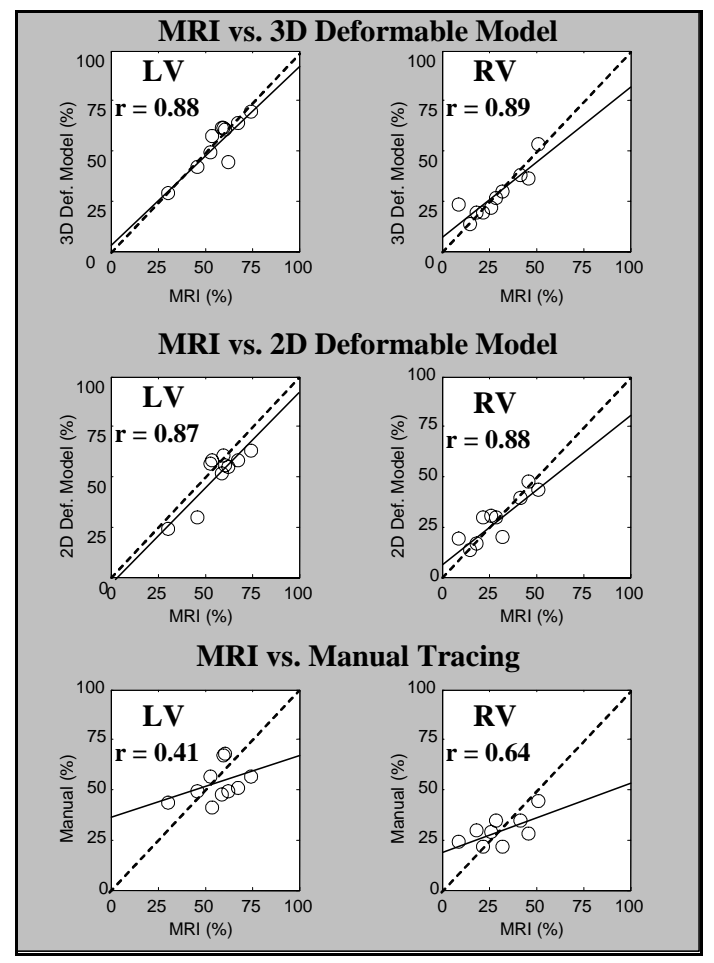

Figure 1: Linear regression for $\mathrm{RV}$ and $\mathrm{LV}$ ejection fraction measures estimated with manual tracing on MRI, manual tracing versus RT3D ultrasound and deformable models on RT3D ultrasound. Correlation coefficients are reported for each case.

For further evaluation, we performed a Bland-Altman statistical analysis [19]. We considered measures from MRI data as the reference (ground truth) and computed errors of RT3D measures vs. MRI [20, 21] with either manual tracing or deformable model. Results of the Bland-Altman analysis are displayed in Figure 2.

The 95\% confidence interval is defined with a center value equal to the mean error and width equal to two standard deviations of the error. Intervals with one standard deviation were equal to:
- 2D DM: $[0.31 \% \pm 6.60 \%]$ for RV EF, and $[5.6 \% \pm 6.42 \%]$ for LV EF.

- 3D DM: $[1.31 \% \pm 6.27 \%]$ for RV EF, and $[2.93 \% \pm 6.13 \%]$ for LV EF.

- Manual tracing: $[1.20 \% \pm 10.09 \%]$ for RV EF, and $[3.93 \% \pm 11.80 \%]$ for LV EF.

Analysis of the errors showed that both 2D and 3D deformable models applied to denoised RT3D ultrasound performed similarly and as accurately as manual tracing while reducing the mean error and the confidence interval by up to $37 \%$ for RV EF and $48 \%$ for LV EF.

Takuma et al. [22] reported standard error of estimate of $4 \%$ for $\mathrm{LV}$ EF measures, when comparing to MUGA. They also reported inter-observer variability of $2.4 \%-3.7 \%$ and intra-observer variability of $3.9 \%$ 8.3\% for two different manual tracing methods.

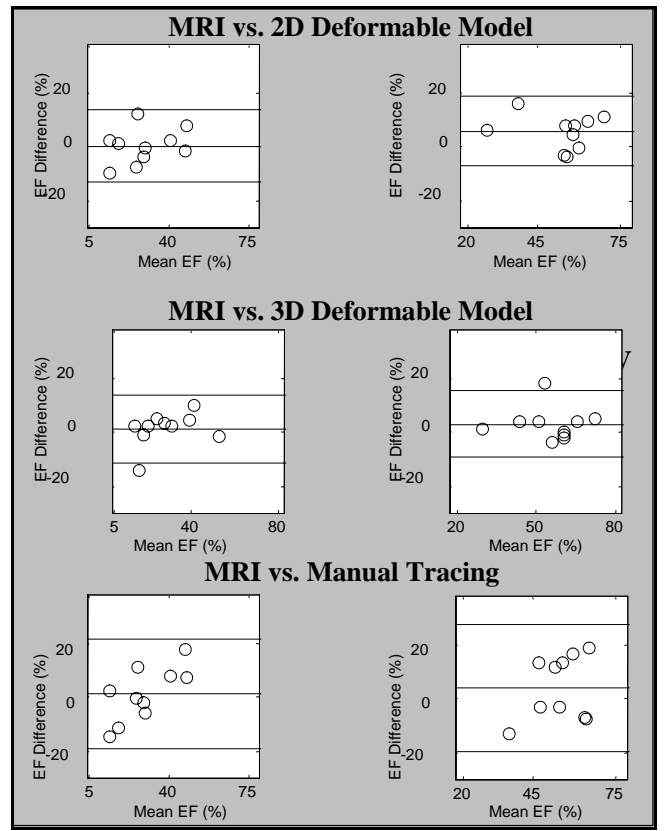

Figure 2: Bland-Altman statistical analysis for $\mathrm{LV}$ and RV EF measures with manual tracing and 2D and 3D deformable model on RT3D ultrasound vs. manual tracing on MRI. Average EF difference and 95\% confidence interval are displayed on each plot.

Our study showed that 2D and 3D deformable model could achieve 6\% accuracy for RV and LV ejection fraction with average errors below inter and intra observer variability reported by similar studies. We demonstrated that three-dimensional deformable model improved LV EF measures, which can be explained by a more accurate segmentation of small and distorted ventricular shapes when integrating the third spatial dimension. We can conclude that quantification of cardiac function with RT3D ultrasound, via deformable model segmentation on denoised data, is feasible and provides accurate volume and ejection fraction measures, compared to MRI. Manual tracing measures were significantly less reliable with large standard 
deviation of errors and yielded significantly lower correlation coefficients.

\section{Conclusion}

We have implemented deformable model segmentation algorithms in 2D and 3D for quantification of ventricular function using RT3D ultrasound. The 2D deformable model was derived from the balloon method while the 3D method was derived from the MumfordShah minimization method, deforming a surface to achieve an optimal partitioning of the data into smooth regions. These deformable models proved to be very well suited for the segmentation of RT3D cardiac ultrasound as they extracted highly curved volumes while ensuring smoothness to handle missing myocardium signals and inside localized echos from trabeculations.

A complete and rigorous validation of the segmentation methods was carried out for quantification of left and right ventricular ejection fraction including comparison of measurements with cardiac MRI as the reference. Errors of measurement for both ventricles were within the range of inter- and intra- observer variability and compared favorably to similar studies performed by other groups using RT3D ultrasound for quantification of cardiac function. Manual tracing measures were significantly less reliable with large standard deviation of errors and low correlation coefficients. Finally, the 3D deformable model achieved the highest degree of accuracy, which can be explained by a more accurate segmentation of small and distorted ventricular shapes when integrating the third spatial dimension.

\section{References}

[1] O. T. Von Ramm and S. W. Smith, "Real time volumetric ultrasound imaging system," Journal of Digital Imaging, vol. 3, pp. 261-266, 1990.

[2] J. A. Sethian, "Theory, algorithms, and applications of level set methods for propagating surfaces," Acta Numerica, pp. 309-395, 1996.

[3] W. J. Niessen, B. M. t. H. Romeny, and M. A. Viergever, "Geodesic deformable models for medical image analysis," IEEE Transactions on Medical Imaging, vol. 17, pp. 634 - 641, 1998.

[4] J. Montagnat, H. Delingette, and N. Ayache, "A review of deformable surfaces: topology, geometry and deformation," Image and Vision Computing, vol. 19, pp. 1023-1040, 2001.

[5] C. Xu, D. L. Pham, and J. L. Prince, "Image segmentation using deformable models," in Handbook of Medical Imaging, Vol. 2: Medical Image Processing and Analysis, vol. 2: SPIE Press, 2000, pp. 131-169.

[6] T. F. Chan and L. A. Vese, "Active contours without edges," IEEE Transactions on Image Processing, vol. 10, pp. 266 - 277, 2001.

[7] D. Mumford and J. Shah, "Boundary detection by minimizing functional," presented at International Conference on Computer Vision and Pattern Recognition, San Francisco, CA, USA, 1985.

[8] S. Osher and J. A. Sethian, "Fronts propagating with curvature-dependent speed: Algorithms based on
Hamilton-Jacobi formulations," Journal of Computational Physics, vol. 79, pp. 12-49, 1988.

[9] H.-K. Zhao, T. Chan, B. Merriman, and S. Osher, "A variational level set approach to multiphase motion," Journal of computational physics, vol. 127, pp. 179-195, 1996.

[10] R. Malladi, J. A. Sethian, and B. C. Vemuri, "Shape modeling with front propagation: A level set approach," IEEE Transactions on Pattern Analysis and Machine Intelligence, vol. 17, pp. 158-175, 1995.

[11] L. I. Rudin, S. Osher, and E. Fatemi, "Nonlinear total variation based noise removal algorithms," Physica $D$, vol. 60, pp. 259--268, 1992.

[12] G. Aubert and L. Vese, "A variational method in image recovery," SIAM Journal on Numerical Analysis, vol. 34, pp. 1948-1979, 1997.

[13] J. A. Sethian, "Fast marching methods," SIAM Review, vol. 41, pp. 199-235, 1999.

[14] M. Sussman, P. Smereka, and S. Osher, "A level set approach for computing solutions to incompressible twophase flow," Journal of Computational Physics, vol. 114, pp. 116-159, 1994.

[15] E. D. Angelini, A. F. Laine, J. Donis, D. Gersony, and S. Homma, "Quantification of right and left ventricular function with real-time three-dimensional ultrasound," presented at IEEE Engineering in Medicine and Biology Society Annual Conference, Istanbul, Turkey, 2001.

[16] E. Angelini, A. Laine, S. Takuma, J. Holmes, and S. Homma, "LV volume quantification via spatio-temporal analysis of real-time 3D echocardiography," IEEE Transactions on Medical Imaging, vol. 20, pp. 457-469, 2001.

[17] E. D. Angelini, "Spatio-Temporal Analysis of ThreeDimensional Real-Time Ultrasound for Quantification of Ventricular Function," Columbia University, New York, Ph.D Thesis 2002.

[18] C. Corsi, M. Borsari, F. Consegnati, A. Sarti, C. Lamberti, A. Travaglini, T. Shiota, and J. D. Thomas, "Left ventricular endocardial surface detection based on real-time 3D echocardiographic data," European Journal of Ultrasound, vol. 13, pp. 41-51, 2001.

[19] J. M. Bland and D. G. Altman, "Statistical methods for assessing agreement between two methods of clinical measurements," Lancet, vol. 1, pp. 307-310, 1986.

[20] T. Shiota, M. Jones, M. Chikada, C. Fleishman, J. Castellucci, B. Cotter, A. DeMaria, O. T. Von Ramm, J. Kisslo, T. Ryan, and D. Sahn, "Real-time three dimensional echocardiography for determining right ventricular stroke volume in an animal model of chronic right ventricular volume overload," Circulation, vol. 97, pp. 1897-1900, 1998.

[21] M. Vogel, M. Gutberlet, S. Dittrich, N. Hosten, and P. E. Lange, "Comparison of transthoracic three dimensional echocardiography with magnetic resonance imaging in the assessment of right ventricular volume and mass.," Heart, vol. 78, pp. 127-130, 1997.

[22] S. Takuma, T. Ota, T. Muro, I. Oropesa, R. Sciacca, L. Mendoza, M. R. D. Tullio, D. K. Blood, J. Yoshikawa, and S. Homma, "Assessment of left ventricular function by real-time 3D echocardiography compared with conventional noninvasive methods," Journal of the American Society of Echocardiography, vol. 14, pp. 275-284, 2001. 